\title{
MAPPING VISUAL FIELDS IN A PANORAMIC DRIVING SIMULATOR UNDER DIFFERENT TASK LOADS IN PATIENTS WITH GLAUCOMA
}

\author{
Deepta Ghate $\mathrm{MD}^{1}$, David Anderson $\mathrm{PhD}^{2}$, Jideofor Ndulue $\mathrm{MD}^{1}$, \\ Robin High MS ${ }^{3}$, Lynette Smith $\mathrm{PhD}^{3}$, Matthew Rizzo $\mathrm{MD}^{2}$ \\ ${ }^{1}$ Truhlsen Eye Institute, ${ }^{2}$ Department of Neurological Sciences, ${ }^{3}$ College of Public Health, \\ University of Nebraska Medical Center, Omaha, NE, USA \\ Email: ghate@unmc.edu
}

\begin{abstract}
Summary: Glaucoma causes visual field loss, which may impair detection of objects and hazards during driving. Standard clinical visual field testing, developed to address status of disease, is not designed to capture the effects of visual field loss in ecological settings. To address this need, we developed a driving stimulus detection task (DSVF) similar to clinical perimetry for deployment in a panoramic driving simulator. The outcome measure is a gray scale map of the driver's response to visual test stimuli in the panoramic driving environment 22 glaucoma subjects and 18 controls completed the DSVF under: a) conditions similar to clinic perimetry with a fixation target; b) a no-driving condition with eye and head movements allowed; and c) while driving. The derived visual field index (DSVFVFI) decreased with increasing task load in both groups, and more so in glaucoma. A predictive formula was generated that allows an estimate of the driver's available field of view under different task loads from clinical perimetry results.
\end{abstract}

Safe driving requires sustained attention to incoming sensory information for timely response to on-road hazards and events and is affected by disorders of the brain and the eye. Glaucoma is an optic neuropathy which causes peripheral vision loss while sparing the central vision in all but end-stage disease. Glaucoma is a major public health concern, affecting $2 \%$ of the total U.S. population over the age of 40 years (Friedman, 2004) and may increase the risk of a motor vehicle crashes (Kwon, 2016). Clinical perimetry is used to map a glaucoma patient's peripheral visual field, most commonly using Humphrey visual fields (HVF). HVF are routinely mapped monocularly with a fixation target under no-distraction conditions.

Patients with glaucoma often ask, "can I drive?" The answer has important implications for the patient's safety, mobility, life-space, and quality of life. Accurate information is needed on what part of the driving environment is visually "available" under realistic, ecologically valid settings, that is not addressed with standard clinical perimetry. In response, we designed a novel driving simulator visual field (DSVF) test to map the field of view in realistic driving conditions. Our aims were to 1) assess the validity and reproducibility of the DSVF task and 2) quantify the "available" field of view in subjects with glaucoma under differing driving task loads. Our hypothesis was that "available" field of view will decrease with increasing driving task load. We propose that DSVF results are of greater relevance to driving than standard clinical perimetry. In particular, the results of the DSVF task may give us a better understanding of patient response to road hazards in an area of compromised visual field. 


\section{METHODS}

This study was performed under the supervision of the University of Nebraska Medical Center (UNMC) Institutional Review Board.

Subjects: All subjects were recruited from the Truhlsen Eye Institute at UNMC in Omaha NE. Inclusion criteria included best corrected visual acuity of at least 20/40 in worse eye and presence of 2 reliable (reliability indices better than 20\%) and reproducible (subjective evaluation by glaucoma specialists) HVF over the last 2 years. Visual fields were performed using the Humphrey field analyzer (Carl Zeiss Meditec Inc) using the 24-2 or 30-2 strategy as part of routine clinical care. Controls had normal visual fields (subjective evaluation and a normal glaucoma hemifield test) with a clinical diagnosis of ocular hypertension or glaucoma suspect. Glaucoma subjects had visual field defects commensurate with their disease. Subjects were excluded if they had a previous diagnosis of neurological disorder or cognitive impairment.

The Driving Simulator visual field (DSVF) scenario was coded and implemented in SENSEI (Simulator for Ergonomics, Neuroscience, Safety Engineering and Innovation), a DriveSafety RS-600 (Salt Lake City, UT), fully integrated, high performance, high fidelity driving simulation system with an authentic automotive cab (based on a 2004 Ford Focus) and an out-the-window display environment of $290^{\circ}$. DSVF tests total $60^{\circ}$ horizontal and $20^{\circ}$ vertical visual field at 2.5 $\mathrm{m}$. Forty grid test locations are placed $6^{\circ}$ apart, straddling the horizontal and vertical meridian similar to HVF 30-2 strategy. Red supra-threshold stimulus images ( $0.5^{\circ}$ visual angle, similar to HFA stimulus size III) are presented randomly 4 times at each locus with stimulus duration 200 milliseconds, and a varying inter-stimulus interval from 1.2 to 1.7 seconds.

All subjects underwent the following tasks in the same order in the driving simulator with successively increasing task load. Each task duration was approximately 4 minutes. All tasks were repeated twice to test for reproducibility. (1) Task1: The DSVF with a fixation target and grey background in the right eye (OD), left eye (OS) and both eyes (OU). This was a no-driving, no distraction task similar to the HVF; (2) Task 2A: DSVF in a no-driving condition with a naturalistic background with unrestricted eye and head movements; (3) Task 2B: DSVF task superimposed on a driving scenario (straight rural road without any other vehicles or turns and a speed limit of 55 miles per hour). The naturalistic background introduces visual information that competes for access to attention resources. The driving condition requires dividing attention resources across two primary task demands (driving and the DSVF task).

HVF-VFI: Visual field index (VFI) estimates the unimpaired proportion of visual field, ranging between $0 \%$ (fully impaired) to $100 \%$ (fully intact). Monocular HVF-VFI values (labelled as $H V F O D$ and $H V F O S$ for right and left eyes) were directly acquired from the HVF. Binocular integrated visual fields (labelled as $H V F-O U$ ) were derived from the right and left HVF for each patient using the binocular summation method described by Nelson-Quigg et al (Nelson-Quigg 2000). VFI calculations for the integrated HVF-OU were performed using standard published technique (Bengtsson and Heijl 2008).

DSVF-VFI: Each locus was tested 4 times in a multisampling suprathreshold algorithm. Pass criterion was defined if the subject responded to more than $50 \%$ of the stimuli on that locus. This 
pass criterion was chosen for its sensitivity and specificity trade-off (Artes 2003). VFI calculations were performed using standard HVF technique of Bengtsson and Heijl (Bengtsson and Heijl 2008) to calculate a DSVF-VFI for each field. The DSVF-VFI was our main outcome measure and gives a percentage estimate of the "available" field of view (tested area with correct response in the stimulus detection task) in the different task conditions.

Statistical analysis: Participant characteristics were compared between groups using t-tests and chi-square tests. Reproducibility of measurements for the glaucoma group was assessed using intraclass correlation coefficients (ICC). ICC values greater than 0.9 indicate high reproducibility. A logit transformation was applied to calculated visual field values for data analysis and then back-transformed for reporting purposes. Generalized linear mixed models (GLMM) were used to analyze the transformed visual field data comparing the between subject effect, diagnosis (control/glaucoma); and the within subject effect, task, ordered by increasing levels of difficulty, adjusting for subject age. Correlation of multiple observations from each subject were accounted for with a random intercept model. Some of the subject data was missing. The extent and reasons for missing data are explained elsewhere in the report. To include subjects with missing values, multiple imputation with appropriate analysis techniques were implemented, given the missing at random assumption seemed reasonable. Statistical analyses were generated with the GLIMMIX, MI, and MIANALYZE procedures from SAS/STAT software. Graphs were produced with the SGPLOT procedure from SAS/BASE software, Version 9.4 (C) 2002 - 2012) of the SAS System for Windows (SAS Institute Inc., Cary, NC).

\section{RESULTS}

This study included 18 controls and 22 glaucoma subjects. The average age (mean \pm SD) of the controls was $60 \pm 13$ years and the glaucoma subjects was $71 \pm 12$ years (t-test, $p=0.01$ ). There was no significant difference in MOCA scores between the 2 groups. There were $72 \%$ women in the control group and $41 \%$ women in the glaucoma group (chi square test, $\mathrm{p}=0.05$ ). Thirty-six $(90 \%)$ of our 40 subjects were Caucasian. The HVF-VFI is the control group of glaucoma suspects ranged from $98-100 \%$ and in the glaucoma subjects ranged from $16-99 \%$ in the worse eye and $19-100 \%$ in the better eye.

\section{a) Validating new DSVF task as compared to clinic HVF:}

Reproducibility: The DSVF task was highly reproducible. In the control group, the mean difference in VFI between the two trials of DSVF was $0.3-1 \%$ in the no-distraction task (task 1) and $0.4 \%$ and $0.9 \%$ respectively in task $2 \mathrm{~A}$ and task $2 \mathrm{~B}$. In the glaucoma subject group, the mean difference in VFI between the two trials of DSVF was $0.1-2 \%$ in task 1 and $1 \%$ in both task $2 \mathrm{~A}$ and task 2B. Intra class correlation (ICC) calculations for the 2 DSVF trials ranged from 0.910.98. Control subjects were omitted from ICC calculations since the very small subject variability produced deceptively smaller ICCs.

Comparison to gold standard HVF: The DSVF gray scale in the no-distraction task was subjectively very similar to the monocular and calculated binocular HVF subjectively (Figure 1). In glaucoma subjects, the VFI calculations of the DSVF (no-distraction task) and HVF 
correspond extremely well (ICC of 0.9 for OD and OS and 0.8 for OU). ICC calculations were not meaningful for the control subjects due to lack of subject variability.

Blind spot mapping and eye tracking data: The blind spot was mapped accurately (15-21 location temporally) in 78/80 monocular DSVFs (Figure 1). Eye tracking data was available for 13 DSVFs and showed excellent fixation throughout task 1 (non-distraction task) (average saccadic distance in the non-distraction task was $4.7+/-2.1$ )

b) A-pillar scotoma: In all DSVF trials (Figure 1), there was a vertical scotoma in the left $21^{\circ}$ 27 location in the DSVF corresponding to the vehicle's A-pillar. This was calculated as (100DSVF-VFI) both monocularly and binocularly in the control group (where the HVF-VFI is 100 by definition since the controls do not have any peripheral vision loss). The A-pillar caused $8 \pm 5$ $\%$ decrease in VFI OD, $9 \pm 3 \%$ decrease in VFI OS and $4 \pm 3 \%$ decrease in VFI OU.

\section{c) VFI change with different task loads:}

Forty subjects completed task1 (one subject completed only one trial), 37 subjects completed task $2 \mathrm{a}$ ( 2 subjects completed only one trial) and 35 completed task $2 \mathrm{~b}$. Of the subjects with incomplete testing, 4 subjects had simulator adaptation syndrome and the others did not choose to continue. The DSVF-VFI significantly decreased with increasing task driving load in both controls $(\mathrm{p}<0.001)$ and glaucoma subjects $(\mathrm{p}=<0.001)$ (Figure $\& 2)$. The VFI decrease from task 1 to task $2 \mathrm{~b}$ is larger for the glaucoma group as compared to the control group $(\mathrm{p}<0.018)$. Age was not significantly associated with change in DSVF-VFI $(p=0.44)$. Figure 1 displays the gray scales from the DSVF output. Figure 2 is the table and graph of the DSVF-VFI values across the 2 groups in task $1,2 \mathrm{a}$ and $2 \mathrm{~b}$.

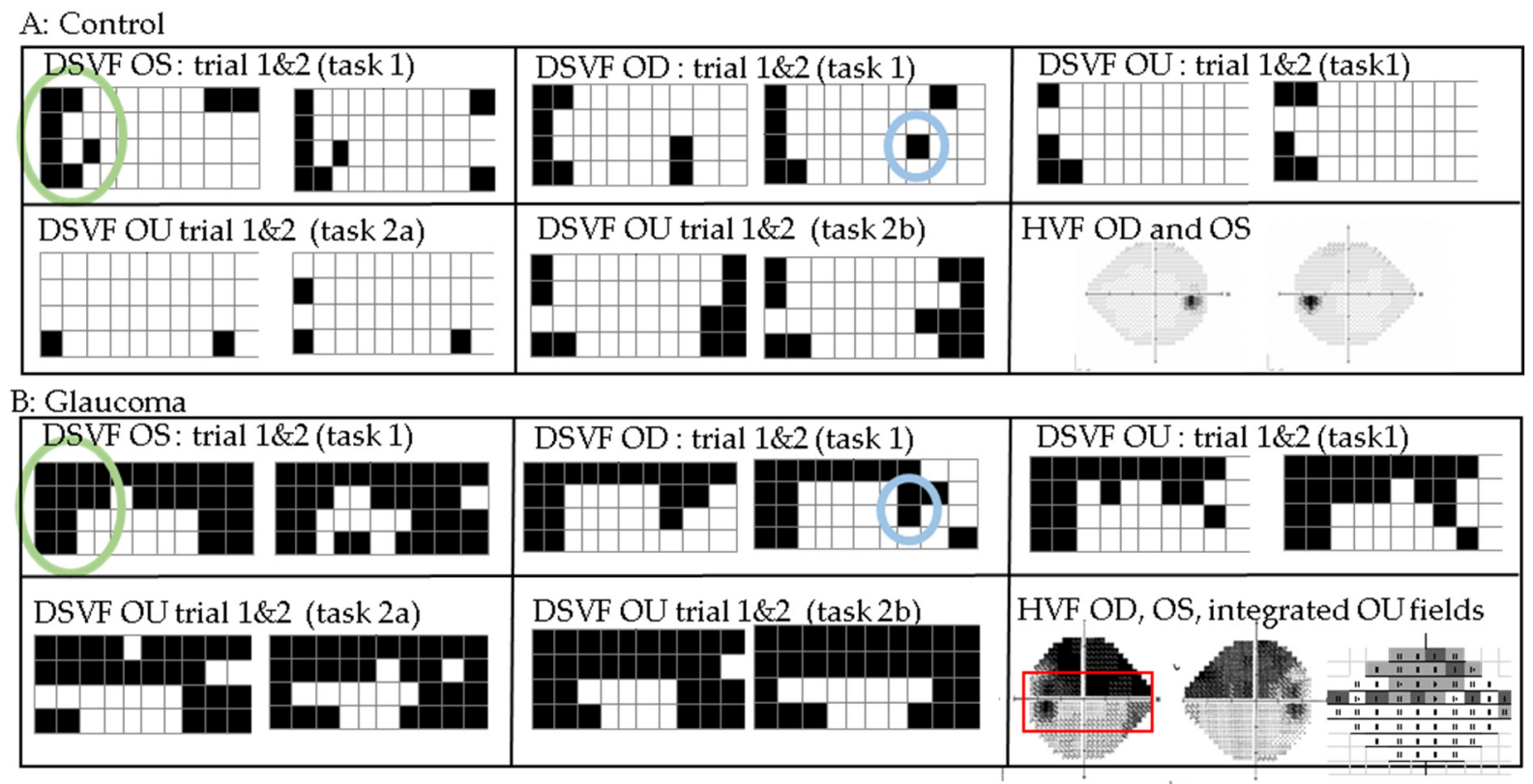

Figure 1: Gray scales of DSVF outputs in controls (A) and glaucoma (B) in tasks 1, 2A and 2B. Each task was repeated twice (trial 1 and 2). Black represents a not-seen locus. Accurate positioning of blind spots (blue circle) is seen in monocular fields and A-pillar scotoma (green circle) is seen in all fields. HVF gray scales demonstrate similarity of gray scales in DSVF and HVF apart from the A-pillar scotoma (green). There is peripheral constriction of visual fields under increasing task load (task $2 b$ ). This constriction is more pronounced in the glaucoma group. 
d) Predictive formula for change in DSVF under different task loads. HVF OU values (from clinic perimetry) were selected as independent variables and a predictive formula was generated using the methodology described above in the 22 Glaucoma subjects. All subjects in the control group had VFI calculated values close to $100 \%$ with little variation among them so they weren't included in the predictive modeling. The predictive formula is displayed in Figure 3

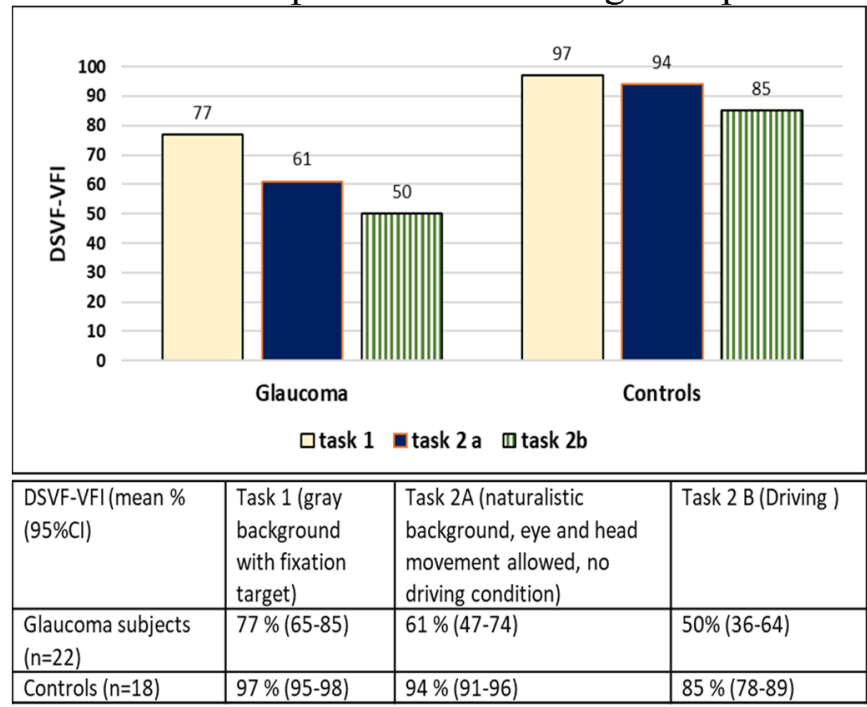

Figure 2: DSVF-VFI values in glaucoma subjects and controls. There is decrease in DSVF-VFI with increasing task load in both glaucoma subjects and controls, the decrease in larger in the glaucoma subject group

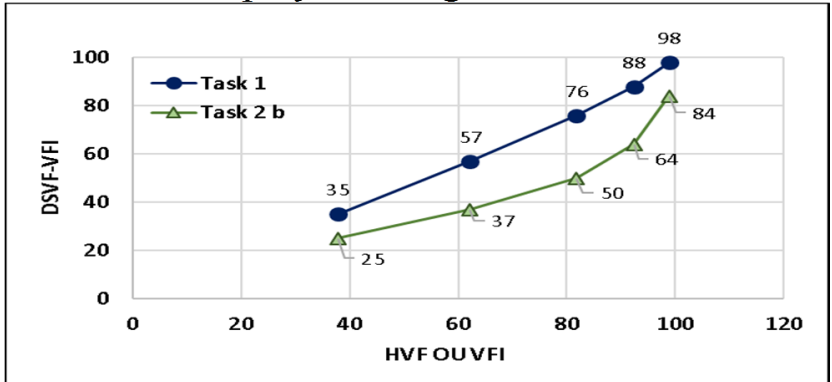

Figure 3: Graph of the predictive DSVF-VFI means at age 70. $x$ axis is binocular calculated HVF OU (from clinic based visual fields). $y$ axis includes task 1 and task 2 b. The predicted DSVF-VFI during task 1 was calculated as $98 \%$ (95\%CI: 94-99), 76\% (95\%CI: 69$82)$ and $35 \%(95 \% C I: 19-55)$ for a subject with $99 \%$, $82 \%$ and $38 \%$ HVF-OU VFI respectively (blue line with circles). The predicted DSVF-VFI in task $2 \mathrm{~b}$ was calculated as 84\% (95\%CI: 63-94), 50\% (95\%CI: 41$60)$ and $25 \%(95 \%$ CI: $13-44)$ for a subject with $99 \%$, $82 \%$ and $38 \%$ HVF-OU VFI respectively (green line with triangles)

\section{DISCUSSION}

The association between visual defects and MVC in glaucoma requires confirmation and further detail (Rubin 2007). Glaucoma may nearly double the risk of an at-fault motor vehicle crash (MVC) (Kwon 2016) depending on degree of field loss.

Subjects: Our study design did not match for age. Despite glaucoma subjects being older than the control group, age was not significantly associated with change in DSVF-VFI. Our predictive formula was calculated using an estimated age of 70. This makes it a particularly valuable tool in assessing field of view in the elderly glaucoma population.

DSVF task: Our DSVF task allows us to evaluate the effect of binocularity, and varying task load on field of view in a naturalistic setting. We eliminated restrictions on head and eye movement to best answer our research question "how does increased task load affect a stimulus detection task in the driving environment". Our visual field methodology (field of view, visual field index, field mapping etc), design (stimulus size, loci) and scoring allow comparison with clinical perimetry. The gray scale should be considered a map that blacks out "missed" areas" of the driver's environment. Our results and past pilot data (Anderson 2016) demonstrate that the DSVF task is reproducible. Accurate blind spot mapping and the similarity of the gray scales to HVF in task 1 demonstrates the validity of the scenario design.

Predictive formula: We developed a predictive formula (Figure 3) to estimate the "available" field of view of a glaucoma patient from their clinic based HVF tests. This predictive formula 
was derived only from the glaucoma group. When the predictive formula was applied to a HVFVFI of 99\% (almost normal HVF), the predicted DSVF values (98\% DSVF-VFI for task 1 and $84 \%$ DSVF-VFI for task $2 \mathrm{~b}$ from figure 3 ) matched those of the control group in our experiment (97\% DSVF-VFI for task 1 and 85\% DSVF-VFI for task 2 b from figure 2 ) which validates the formula.

A-pillar scotoma: Physical obstruction due to in-cab geometry can be mapped out accurately using the DSVF task. Vargas-Martin (Vargas-Martin 2005) have previously taken wide-angled photographs from a camera on the driver's spectacles and noted the size of obstructive in-cab elements relative to the driver's view. Our predictive formula (figure 3) demonstrates that the Apillar scotoma does not vary with worsening glaucoma.

Visual field loss with increasing task load: Gangedulla et al (Gangedulla 2017) and Park et al (Park 2014) have studied the effect of task load on a stimulus detection task and found that the addition of cognitive tasks (steering and driving) decreased the field of view in subjects with glaucoma and controls. The Park study was not conducted in a driving simulator and the Gangedulla study was conducted on a desktop simulator, both with a visual array that doesn't correspond to HVF. The useful field of view (UFOV) task also evaluates the effect of attention on a stimulus recognition task and has been associated with driving performance (Rubin 2007). The UFOV gives a non-spatially specific measure of the role of the attention on stimulus recognition. The strengths of our study include a hi-fidelity driving simulator (full-sized vehicle and wrap around visual displays) and the design of our DSVF task which allows us to map out regions of visual field that are less sensitive to visual information during driving load and compare the output directly to the HVF.

Our study showed that the DSVF-VFI decreased with increasing task load in both the control and glaucoma group. The VFI reductions from task 1 to task $2 \mathrm{~b}$ were greater in the glaucoma group as compared to the control group (figure 2). The predictive formula (figure 3) using only glaucoma subjects demonstrates the same finding. At nearly normal VFIs (very early glaucoma), the driving DSVF-VFI was almost identical to that of the control group but at HVF-VFI of 9095\% (still considered mild glaucoma), there was a sharp decrease in the DSVF performance in task $2 \mathrm{~b}$ as compared to task 1 . This suggests that driving task load related declines in VFI resulted from additional cognitive factors. Numerous studies of dual task interference have demonstrated that visual search is slowed under additional task demands (Han 2004). Taken together, these results suggest glaucoma patients may also be impaired in completing multiple task demands, such as driving and visual search.

Our study aims to assist clinicians predict driving performance based on HVF. We believe that our study provides the missing link between clinic-based HVF and on road driving performance by mapping out the DSVF under different driving task loads. Using the predictive formula, a clinician can predict that a patient with moderate glaucoma and a binocular VFI of $82 \%$ will have 76\% VFI in task 1 (A-pillar scotoma will cause a 6\% drop in VFI) and 50\% VFI in task 2 (while driving, the patient will have a further drop of 32\% in VFI). The effect of the A-pillar scotoma is of particular relevance to monocular patients and those with severe glaucoma. For example, a patient with a blind right eye and severe glaucoma (VFI 15\%) in the left eye will lose an additional $9 \%$ of his tested visual field while driving due to the A-pillar scotoma. Future 
research directions will involve correlating HVF and DSVF performance to simulator and onroad driving performance.

\section{CONCLUSION}

The DSVF is a novel driving simulator task that allows us to map out the part of the driving environment that is "missed" under conditions of divided attention and to quantify the extent of this visual field constriction in subjects with and without vision loss. Subjects with glaucoma have a larger decrease in the "available" field of view with increasing task load as compared to controls. Impaired driving performance in glaucoma subjects is likely a function of both their constricted peripheral vision and reduced ability to efficiently distribute attention across competing task demands. Our results could potentially lead to the development of vehicular alerts, when obstacles appear areas of uncompensated field defects, similar to blind spot alert systems.

\section{ACKNOWLEDGEMENTS}

Funding source: National Institute of General Medical Sciences, 1U54GM115458-02, and by NIA R01 AG017177. The content is solely the responsibility of the authors and does not necessarily represent the official views of the NIH. With thanks to Madeline Sharp for patient recruitment and to our subjects.

\section{REFERENCES}

Anderson, D. E., Ghate, D. A., Kedar, S., \& Rizzo, M. (2017). A New Method for Estimating Effects of Visual Field Loss in a Panoramic Driving Environment. Proceedings of the Ninth International Driving Symposium on Human Factors in Driver Assessment, Training, and Vehicle Design, 2-8.

Artes PH et al (2003). Multisampling suprathreshold perimetry: a comparison with conventional suprathreshold and full-threshold strategies by computer simulation. Invest Ophthalmol Vis Sci. 44(6):2582-7

Bengtsson, B., \& Heijl, A. (2008). A Visual Field Index for Calculation of Glaucoma Rate of Progression. American Journal of Ophthalmology, 145(2), 343-353.

Friedman DS et al (2004). Eye Diseases Prevalence Research Group. Prevalence of open-angle glaucoma among adults in the united states. Arch Ophthalmol. 122(4): 532-538.

Gangeddula V et al (2017) . Effect of Cognitive Demand on Functional Visual Field Performance in Senior Drivers with Glaucoma. Front Aging Neurosci. ;9:286.

Han SH, Kim MS(2004). Visual search does not remain efficient when executive working memory is working. Psychol Sci.;15(9):623-8.

Kwon, $\mathrm{M}$ et al (2016). Association between Glaucoma and At-fault Motor Vehicle Collision Involvement among Older Drivers. Ophthalmology, 123(1), 109-116.

Nelson-Quigg, Jet al (2000). Predicting binocular visual field sensitivity from monocular visual field results. Investigative Ophthalmology \& Visual Science, 41(8), 2212-21.

Park GD, Reed CL (2014) . Nonuniform changes in the distribution of visual attention from visual complexity and action: A driving simulation study. Perception, 43,129-144

Rubin GS et al(2007).A prospective, population-based study of the role of visual impairment in motor vehicle crashes among older drivers: SEE study.Invest Ophthalmol Vis Sci.1483-91

Vargas-Martín F et al (2005). Visual fields at the wheel. Optom Vis Sci. 82(8):675-81. 